\title{
Correlación del umbral de potencial evocado auditivo de tronco cerebral con el umbral de potencial evocado auditivo de estado estable en pacientes hipoacúsicos
}

\author{
Correlation of the threshold of auditory brainstem response \\ with the auditory steady state response in hearing loss patients
}

\author{
Alberto José Guillén $\mathbf{M}^{1}$, Juan Bautista Calero $\mathrm{D}^{2}$, Inmaculada Martínez $\mathrm{A}^{3}$, \\ Francisco García-Purriños G4.
}

\begin{abstract}
RESUMEN
Introducción: El avance de los dispositivos de ayuda auditiva han ocasionado a su vez el desarrollo de las técnicas electrofisiológicas destinadas al diagnóstico de la sordera.

objetivo: Comparar los umbrales del potencial evocado auditivo de tronco cerebral (PEATC) y del potencial evocado auditivo de estado estable (PEAee) en niños con hipoacusia.

Material y método: Estudio observacional descriptivo de una serie de casos de pacientes de 0 a 6 años de edad con hipoacusia, diagnosticada mediante PEATC y PEAee. Se compara el umbral de la onda $V$ en el PEATC y la media del valor registrado en 2 y 4 $\mathrm{kHz}$ en el PEAee mediante la correlación de Spearman y se utiliza el índice Kappa para conocer la concordancia entre las mismas.

Resultados: Se diagnosticaron 89 oídos con hipoacusia, 23 con hipoacusia profunda. Se obtiene un valor de rho de Spearman de 0,64 $(p<0,001)$. El índice de Kappa obtenido es del 0,59 con un IC al 95\% entre 0,52 y 0,66 ( $p<0,001)$. La diferencia media de umbrales entre el PEAee y el PEATC es de -2,42 dB con una desviación estándar de 13,11 dB.

Conclusión: Se obtiene un grado de relación y acuerdo moderado entre las pruebas, influido sobre todo por los pacientes con hipoacusia profunda. Los umbrales del PEAee son mayores que los del PEATC.

Palabras clave: Potenciales de acción, potenciales evocados auditivos, tronco cerebral, células receptoras sensoriales

\footnotetext{
Servicio de Otorrinolaringología, Hospital General Universitario Santa Lucía, Cartagena, España.

2 Servicio de Otorrinolaringología, Hospital Perpetuo de Socorro, Cartagena, España. Grupo de investigación en Cabeza y Cuello de la Universidad Católica de San Antonio (UCAM), Murcia, España.

3 Servicio de Pediatría, Sección de Neuropediatría, Hospital General Universitario Santa Lucía, Cartagena, España.

4 Servicio de Otorrinolaringología, Hospital Universitario Los Arcos del Mar Menor, Murcia, España. Grupo de investigación en Cabeza y Cuello de la Universidad Católica de San Antonio (UCAM), Murcia, España.
}

Los autores declaran no tener conflictos de interés.

Recibido el 30 de marzo, 2019. Aceptado el 7 de mayo, 2019. 


\section{ABSTRACT}

Introduction: The advancement of hearing aid devices has led to the development of electrophysiological techniques for the diagnosis of hearing loss.

Aim: To compare the thresholds of auditory brainstem response (ABR) with the auditory steady state response (ASSR) in hearing loss children.

Material and method: A descriptive observational study of cases from 0 to 6 years of age with hearing loss, diagnosed by ABR and ASSR was performed. The Spearman correlation test is used to compare the threshold obtained for the $V$ wave in the $A B R$ and the average value recorded in 2 and $4 \mathrm{kHz}$ in the ASSR. To know the concordance between the two tests, the Kappa index is used.

Results: 89 ears were diagnosed with hearing loss, 23 with profound hearing loss. The Spearman's rho value is $0.64(p<0.001)$. The Kappa index obtained is 0.59 with a $95 \% \mathrm{Cl}$ between 0.52 and $0.66(p<0.001)$. The mean difference thresholds between the $A S S R$ and the $A B R$ is $-2.42 d B$ with a standard deviation of $13.11 \mathrm{~dB}$.

Conclusion: A moderate relationship and agreement between the tests is obtained, influence especially in patients with profound hearing loss. The thresholds of ASSR are higher than those of $A B R$.

Key words: Actions potentials, evoked potentials, auditory, brainstem, sensory receptor cells.

\section{INTRODUCCIÓN}

El principal método para el estudio de la audición en humanos es la prueba que se denomina "audiometría por respuesta eléctrica", que cuantifica y cualifica la actividad generada por el sistema nervioso auditivo central, en el tronco, como una respuesta a la estimulación acústica de manera inocua y sin la necesidad de participación del paciente de una forma activa. La obtención y registro de esta respuesta de actividad cerebral se denomina "potencial evocado auditivo de tronco cerebral" (PEATC) que corresponde a fluctuaciones de voltaje en el tiempo generados en el sistema nervioso en respuesta a un estímulo adecuado ${ }^{1}$.

EI PEATC es una prueba electrofisiológica objetiva pero que necesita una interpretación por parte del explorador para distinguir en el trazado eléctrico las diferentes ondas que componen las etapas de la vía auditiva. Desde su desarrollo se ha utilizado para proporcionar información sobre el estado auditivo en pacientes que son demasiado jóvenes o que se encuentran en un nivel de desarrollo en el que no es posible realizar mediciones fiables de la respuesta conductual ${ }^{2}$.

Las características del registro del PEATC se compone de la obtención de un trazado de ondas, siendo las más frecuentemente medidas las denominadas I, II, III, IV y V. Estas ondas presentan características fundamentales de amplitud, que marca la presencia de la respuesta y es variable de unos individuos a otros y disminuyen con la menor intensidad del estímulo; de latencia, que es el tiempo de aparición de una determinada onda desde que se produce el estímulo y aumenta en todas las ondas a medida que disminuye el mismo; y de interlatencia, que es el intervalo de tiempo entre dos ondas, siendo las más importantes la I-III, la III-V y la I-V. EI PEATC nos ofrece información del tipo de hipoacusia y se considera el umbral auditivo la última presencia de la onda $V$ en amplitud y latencia ${ }^{3,4}$ (Figura 1). El PEATC es la principal herramienta electrofisiológica del diagnóstico de hipoacusia, siendo la audiometría conductual la prueba gold standard.

El potencial evocado auditivo de estado estable (PEAee) es una respuesta provocada por la amplitud sinusoidal y/o tonos modulados en frecuencia. Siendo una herramienta más para la evaluación de la audición en niños ${ }^{5,6}$ (Figura 2).

Entre las ventajas del PEAee encontramos varias: el estímulo es multifrecuencial ya que aplica tonos modulados en frecuencias de 0,5, 1, 2, 4 


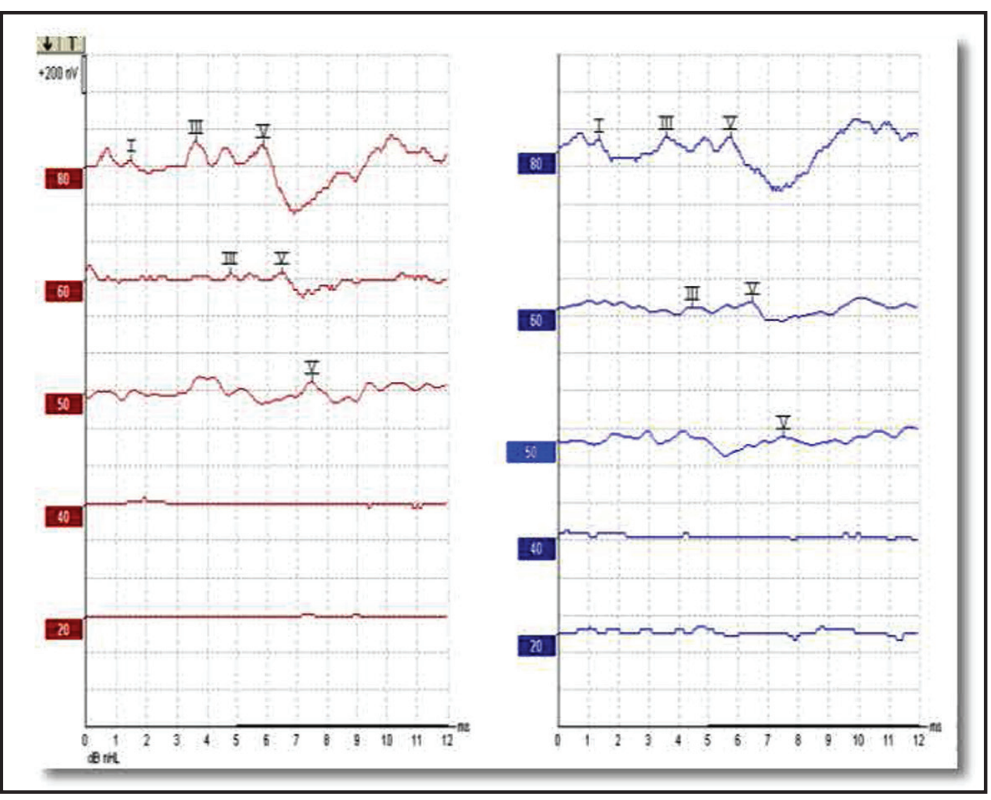

Figura 1. Potencial evocado auditivo de tronco cerebral. PEATC realizados con clics alternantes con resultado de hipoacusia neurosensorial bilateral moderada (umbral a $50 \mathrm{dBs}$ bilateral). La imagen muestra una disminución de la onda I, intervalos interpico I-III, I-V y latencia de onda V normal a altas intensidades y un aumento de la latencia onda $\mathrm{V}$ a bajas intensidades hasta $50 \mathrm{~dB}$ en los dos oídos.

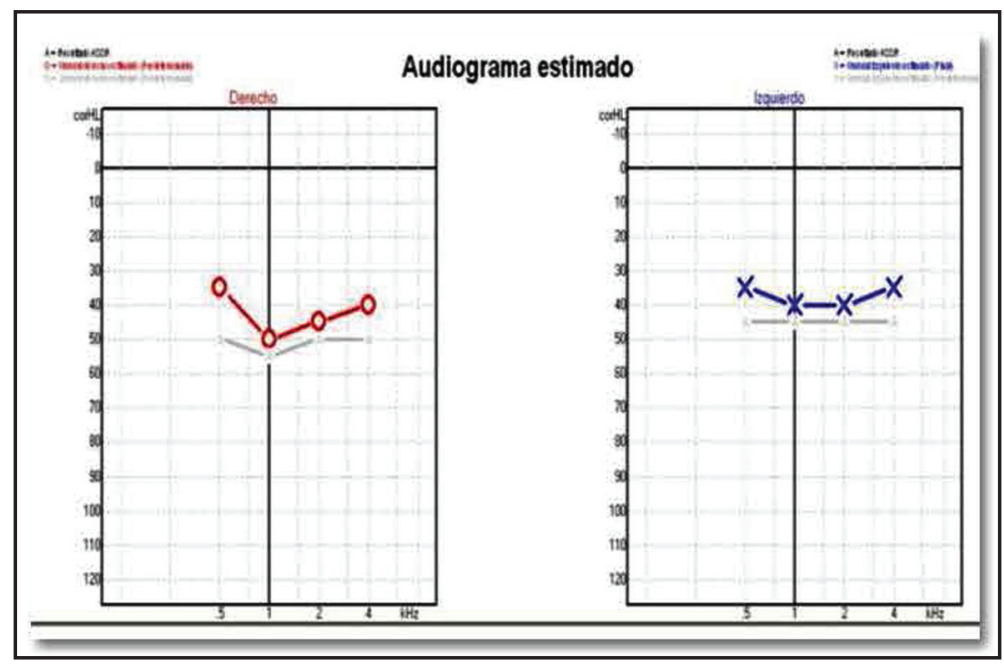

Figura 2. Potencial evocado de estado estable. PEAee con resultado de hipoacusia bilateral moderada. El audiograma muestra Ios valores registrados y estimados en el mismo paciente del PEATC de la Figura 1, con un umbral sobre 40 dB para cada oído.

$\mathrm{kHz}$, en cambio en el PEATC se aplica el estímulo únicamente sobre frecuencias agudas (2 kHz). En el PEAee la respuesta es automática y no necesita interpretación, por lo que evita problemas asocia- dos con la experiencia del explorador. Además, las mediciones del PEAee tienen un rango dinámico más amplio que las mediciones del PEATC, gracias a la larga duración del estímulo, por lo que se 
pueden alcanzar niveles más altos de umbrales en comparación con los estímulos de corta duración del PEATC .

El diagnóstico precoz de la pérdida auditiva en niños y el avance de los dispositivos de ayuda auditiva permiten el desarrollo del habla, el lenguaje y las habilidades necesarias para la comunicación oral. La reducción de la edad mínima de implantación coclear y el desarrollo de los mismos en los últimos años ha aumentado el número de pacientes que requieren de una audiometría objetiva precisa para determinar el manejo de los mismos, por lo que es fundamental conocer cómo funcionan estas dos pruebas de diagnóstico y la detección temprana de una pérdida auditiva.

\section{OBJETIVO}

Los objetivos son comparar los umbrales del PEATC y del PEAee en niños con diferente grado de hipoacusia y discutir las ventajas y desventajas de ambos métodos.

\section{MATERIAL Y MÉTODO}

Se realiza un estudio observacional descriptivo de una serie de casos de 0 a 6 años de edad, en el periodo de tiempo entre enero de 2013 y diciembre de 2017, a los que se realiza PEATC y PEAee mediante auriculares de inserción. Los criterios de inclusión fueron pacientes con diagnóstico de hipoacusia neurosensorial o mixta derivados desde el programa de cribado auditivo neonatal con necesidad de realizar pruebas diagnósticas adicionales y aquellos que tenían algún factor de riesgo de pérdida auditiva independientemente del resultado del cribado. También incluimos a niños que fueron derivados del servicio de pediatría o de nuestro propio servicio para estudio del umbral auditivo debido a su edad y dada la imposibilidad para estudio mediante pruebas conductuales. Los criterios de exclusión fueron no tener alguna de las dos pruebas realizadas, tener más de 6 años de edad y tener ambas pruebas con diagnóstico de normalidad o con diagnóstico de hipoacusia de transmisión.

A todos los niños se les realizó un examen mediante otoscopía, impedanciometría y estudio me- diante PEATC y PEAee (Eclipse, Interacoustics ${ }^{\circledR}$ ). Las pruebas electrofisiológicas fueron realizadas en una sala tranquila, donde el nivel de sonido era inferior a $50 \mathrm{~dB}$ y fueron evaluadas por el mismo médico. Cada niño estaba dormido mediante sueño fisiológico o con hidrato de cloral durante la prueba. El PEATC se realizó mediante estímulos tipo clics con enmascaramiento contralateral con ruido blanco a $-30 \mathrm{~dB}$ y el PEAee mediante tonos puros modulados, simultáneo en ambos oídos para las frecuencias de 0,5, 1, 2, $4 \mathrm{kHz}$.

Para el análisis estadístico se utilizó el programa IBM ${ }^{\odot}$ Statistical Package for the Social Sciences $(\text { SPSS })^{\odot}$ versión 24 para OS $X^{\odot}$. Se realiza un estudio descriptivo de los resultados obtenidos según las pruebas. Para estudiar la relación de los umbrales de audición del PEATC y PEAee se realiza la prueba de correlación de Spearman previa valoración de la bondad del ajuste mediante la prueba de Kolmogorov-Smirnov. Se compara el umbral obtenido para la onda $V$ en el PEATC y la media del valor registrado en 2 y $4 \mathrm{kHz}$ en el PEAee. Para conocer el valor de acuerdo o concordancia entre las dos pruebas también se utiliza el índice Kappa, clasificando el umbral en tres grupos según la gravedad de la hipoacusia en leve, moderada y severa/profunda.

\section{RESULTADOS}

Se incluyeron en el estudio 47 pacientes diagnosticados con algún grado de hipoacusia de 375 pacientes de 0 a 6 años a los que se realizaron ambas pruebas durante los años de estudio. De los 47 pacientes, 30 eran varones (64\%) y 17 mujeres $(36 \%)$. La mediana de edad en el momento del diagnóstico fue de 6 meses con un rango intercuartílico (RIQ) de 3,5 meses. El rango de edad de los pacientes diagnosticados de hipoacusia fue de 1 a 24 meses (Figura 3).

De los 47 niños diagnosticados de hipoacusia existían 89 oídos patológicos. La clasificación de los 89 oídos patológicos según la gravedad de la hipoacusia se muestra en la Figura 4.

Se observa que 6 de los 23 oídos (26\%) de los clasificados como hipoacusia profunda tenían un PEATC que evocaban un potencial, pero con umbrales de hipoacusia profunda y respondían en el 
PEAee en, al menos, dos frecuencias. Sin embargo, 17 de los 23 oídos (74\%) de los clasificados como hipoacusia profunda no evocaban ningún potencial en el PEATC. De éstos, 5 oídos tampoco respondían en el PEAee en ninguna frecuencia, pero 12 oídos sí que respondían en alguna de las frecuencias de la prueba como se muestra en la Figura 5.

Al comparar el umbral de la onda V del PEATC y la media del valor registrado del PEAee se obtiene un valor de rho de Spearman de 0,64, siendo este valor estadísticamente significativo $(p<0,001)$. El

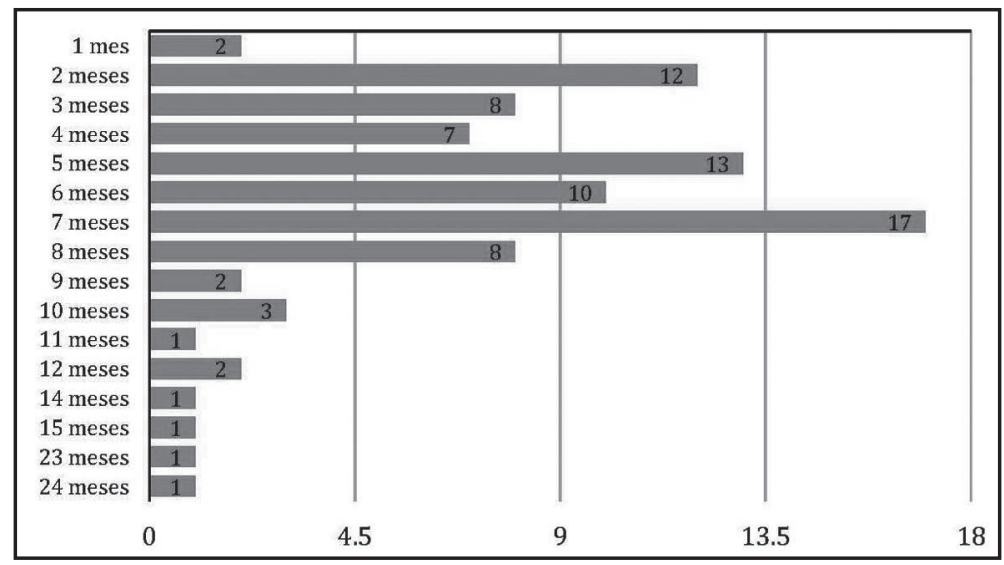

Figura 3. Edad en el momento del diagnóstico.

\section{Hipoacusia leve}

Hipoacusia moderada

Hipoacusia profunda
Hipoacusia severa

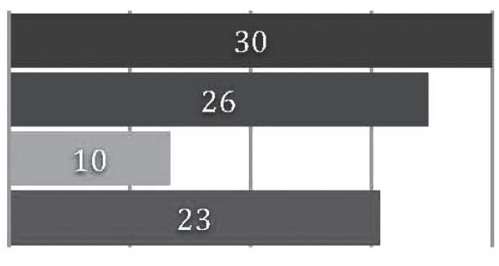

Figura 4. Clasificación de los oídos patológicos según la gravedad de la hipoacusia.

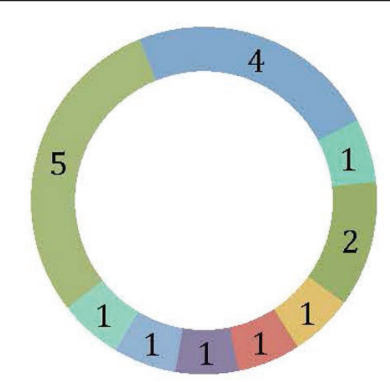

Responde a $0.5,1,2$ y $4 \mathrm{kHz}$

- Responde a $0.5,1$ y $2 \mathrm{kHz}$

Responde a $0.5,1$ y $4 \mathrm{kHz}$

Responde a 0.5 y $4 \mathrm{kHz}$

- Responde a 1 y $2 \mathrm{kHz}$

Responde a 0.5 y $2 \mathrm{kHz}$

- Responde a $2 \mathrm{kHz}$

Responde a $4 \mathrm{kHz}$

No responden en PEAee

Figura 5. Pacientes con hipoacusia profunda no evocaban ningún potencial de acción en el PEATC y su respuesta en PEAee. 
índice de Kappa obtenido es del 0,59 con un IC al $95 \%$ entre 0,52 y $0,66(p<0,001)$ (Tabla 1$)$. La diferencia media de umbrales entre el PEAee y el PEATC fue de -2,42 dB con una desviación estándar de $13,11 \mathrm{~dB}$.

\section{DISCUSIÓN}

El avance en los últimos años de indicaciones cada vez más tempranas de implantación y de uso de dispositivos de ayuda auditiva ocasiona que las pruebas electrofisiológicas que utilizamos tienen que ser precisas para detectar a un paciente hipoacúsico y el umbral auditivo que éste presenta.

La estimulación auditiva para la obtención del registro de los PEATC y los PEAee se realiza habitualmente mediante auriculares de inserción (en el interior del conducto auditivo externo) y el empleo de electrodos de superficie convenientemente colocados $^{8}$. La combinación de ambos registros nos va a permitir obtener umbrales de audición en intensidad y frecuencia, necesarios para el diagnóstico de la hipoacusia infantil. Gracias al programa de cribado implantado en nuestra área, el diagnóstico de la mayoría de nuestros pacientes se realizó en los primeros meses de vida, cuando la implantación coclear adquiere un mayor éxito de adaptación, sin embargo, hubo niños diagnosticados a partir del primer año hasta los dos años de hipoacusia profunda.

EI PEATC nos ofrece información del tipo de hipoacusia y del umbral auditivo gracias a la última presencia de la onda $\mathrm{V}$ en amplitud y latencia. La prueba se considera normal cuando se detecta a $20 \mathrm{~dB}^{4}$. Los estímulos son muy breves, pudiendo emplearse clics, clics filtrados o tone burst. Nosotros utilizamos estímulos tipo clics alternantes con enmascaramiento contralateral. Los criterios de normalidad de los PEAee no precisan una interpretación detallada ya que una vez realizada la estimulación y obtenida una respuesta estable, el software del equipo realiza el algoritmo de transformada de Fourier que es un registro de la respuesta electroencefalográfica correspondiente a la frecuencia modulada del tono presentado y calcula una audiometría estimada con frecuencias de 0,5, 1, 2 y $4 \mathrm{kHz}^{9}$. Se acepta que la obtención de esta respuesta debe aparecer en un tiempo determinado, no superior a los 8-10 minutos.

De los 23 pacientes diagnosticados con hipoacusia profunda, 12 no tuvieron respuesta en el PEATC pero sí en alguna frecuencia de los PEAee. Esta situación nos indica que la determinación de los umbrales para 0,5, 1, 2, $4 \mathrm{kHz}$ en la detección de hipoacusia profunda es fundamental, ya que podemos detectar audición residual en pacientes mediante el PEAee que el PEATC no llega a determinar nunca. Además, el PEATC está limitado a 90-100 $\mathrm{dB}$ de intensidad máxima y el PEAee a $120 \mathrm{dBs}$. De esta manera podemos aplicar un tratamiento inmediato con prótesis auditivas no implantables mientras el paciente se encuentra a la espera de un implante coclear, permitiendo así el mantenimiento de la plasticidad neural auditiva.

Por tanto, mientras que los PEATC con umbrales ausentes no pueden determinar restos auditivos que implicaría audición utilizable, los umbrales de PEAee ausentes indican que no hay audición utilizable. La identificación temprana de niños con una evaluación auditiva precisa lleva a una adaptación más temprana de los dispositivos de ayuda, incluidos los implantes cocleares, que minimizan los retrasos en el desarrollo de la audición y del lenguaje ${ }^{10}$.

El desafío del diagnóstico exacto es cada vez menor gracias a la combinación de estas pruebas.

Tabla 1. Clasificación por grupos según la gravedad de la hipoacusia y pruebas

\begin{tabular}{|lccccc|}
\hline & & & PEATC onda V & \\
& & $<0$ & $41-69$ & $>70$ & Total \\
\hline & $<40$ & 22 & 9 & 0 & 31 \\
PEAee & $41-69$ & 7 & 4 & 3 & 31 \\
(media de & $>70$ & 1 & 34 & 22 & 27 \\
2 y $4 \mathrm{kHz})$ & Total & 30 & & 25 & 89 \\
\hline
\end{tabular}


Las publicaciones hasta la fecha observan que las complicaciones relacionadas con la anestesia y la cirugía son mínimas y la implantación temprana representa un riesgo mínimo para los niños menores de un año de edad. Con ello se obtienen mayores beneficios en áreas de desarrollo del lenguaje receptivo y expresivo y la percepción del habla. Los datos sugieren que la implantación temprana puede maximizar la capacidad de un niño para alcanzar su máximo potencial con el mínimo riesgo asociado, por lo que un diagnóstico temprano y preciso ayuda a la mejor evolución del niño hipoacusico ${ }^{11}$.

Al estudiar la correlación entre los umbrales de la onda $V$ del PEATC y la media obtenida en 2 y $4 \mathrm{kHz}$ en PEAee se obtiene un valor de 0,64, siendo una relación positiva moderada y un grado de acuerdo moderado. Este valor está acorde con otros artículos en la literatura para niños con cualquier grado de hipoacusia ${ }^{11}$, aunque el tamaño de la muestra es menor. En otros artículos el grado de correlación es alto, pero incluyen niños normooyentes junto con los hipoacúsicos ${ }^{10,13,14}$. Igual que desciende si sólo se aplica en hipoacúsicos profundos ya que hay valores que se pierden en ambas pruebas ${ }^{15}$.

\section{BIBLIOGRAFÍA}

1. Trinidad G, Trinidad G, De la Cruz E. Potenciales Evocados Auditivos. An Pediatr Contin 2008; 6 : 296-301.

2. Jewett L, Romano MN, Williston JS. Auditory Evoked Potencials: Possible Brain Stem Components Detected on the Scalp 1970; 167: 1517-18.

3. Rivera S, Marcotti A. Comparación de las amplitudes y latencias de la onda $V$ del potencial evocado auditivo de tronco cerebral obtenidas a través de estímulos clic y CE-Chirp. Rev Otorrinolaringol Cir Cabeza Cuello 2016; 76: 272-9.

4. Guilhoto L, Quintal V, da Costa M. Brainstem auditory evoked response in normal term neonates. Arq Neuropsiquiatr 2003; 61: 906-8.

5. Rickards FW, Tan LE, Cohen LT, Wilson OJ, Drew JH, CLaRK GM. Auditory steady-state evoked
Por último, los resultados de este estudio demuestran que los umbrales de PEAee son superiores a los obtenidos mediante PEATC para la evaluación del nivel de audición ${ }^{7}$, sin embargo no hay que olvidar que el PEATC es una prueba fundamental para proporcionar información del estado de la vía auditiva y el tipo de hipoacusia. Para nosotros, a pesar de las ventajas, utilizar el PEAee de manera aislada no tiene sentido. Por ello pensamos, al igual que numerosos autores, la necesidad de utilizar los PEATC y los PEAee de forma conjunta ${ }^{12,16-19}$. Son dos pruebas fundamentales para proporcionar el umbral de audición del paciente hipoacúsico y así lograr una mayor precisión en la detección temprana de la pérdida auditiva.

\section{CONCLUSIÓN}

El grado de relación y acuerdo entre ambas pruebas es moderado, influido sobre todo por los pacientes con hipoacusia profunda. Los umbrales del PEAee son mayores que los obtenidos mediante PEATC. El PEAee nos permite determinar audición residual en pacientes con hipoacusia profunda.

potential in newborns. Br J Audiol 1994; 28 : 327-37.

6. Lins OG, PICton TW. Auditory steadystate responses to multiple simultaneous stimuli. Electroencephalography and Clinical Neurophysiology 1995; 96: 420-32.

7. Martinez-Beneito P, Morant Ventura A Pitarch Ribas MI, Garcia Callejo FJ, Marco Algarra J. Potenciales evocados auditivos de estado estable a multifrecuencias como técnica de determinación de umbrales auditivos. Acta Otorrinolaringol Esp 2002; 53: 707-17.

8. Calero JB, Guillén AJ, García-Purriños F. Search for normality criteria of auditory brain responses and auditory steady state response with freefield stimulation. Acta Otorrinolaringol Esp 2018; S0001-6519(18)30127-4. DOI: 10.1016/j. otorri.2018.06.005.

9. Werner LA, Folsom RC Mancl LR. The relationship between auditory brainstem response latencies 
and behavioral thresholds in normal hearing infants and adults. Hear Res 1994; 77: 88-98.

10. Firszt JB, Gaggl W, Runge-Samuelson CL, Burg LS, WACKYM PA. Auditory sensitivity in children using the auditory steady-state response. Arch Otolaryngol Head Neck Surg 2004; 130: 536-40.

11. Roland JT, Cosetti M, Wang KH, Immerman S, WaLtzMAN SB. Cochlear implantation in the very young child: Long-term safety and efficacy. Laryngoscope 2009; 119: 2205-10.

12. Rodrigues GR, Lewis DR, Fichino SN. Steadystate auditory evoked responses in audiological diagnosis in children: a comparison with brainstem evoked auditory responses. Braz $J$ Otorhinolaryngol 2010; 76: 96-101.

13. François M, Dehan E, Carlevan M, Dumont H. Use of auditory steady-state responses in children and comparison with other electrophysiological and behavioral tests. Eur Ann Otorhinolaryngol Head Neck Dis 2016; 133: 331-5.

14. Swanepoel DW, Ebrahim S. Auditory steady-state response and auditory brainstem response thresholds in children. Eur Arch Otorhinolaryngol 2009; 266: 213-9.
15. Kandogan T, Dalgic A. Reliability of Auditory Steady-State Response (ASSR): Comparing Thresholds of Auditory Steady-State Response (ASSR) with Auditory Brainstem Response (ABR) in Children with Severe Hearing Loss. Indian $J$ Otolaryngol Head Neck Surg 2013; 65: 604-7.

16. Sainz M. Potenciales evocados auditivos de tronco cerebral. En Manrique, M. y Marco, J. Audiología. Madrid Proyectos Editoriales, S.A. 2014; 147-58.

17. Korzack P, Smart J, Delgado R, Strobel tM, BradFord C. Auditory Steady-State Responses. J Am Acad Audiol 2012; 23: 146-70.

18. Lins OG, Picton TW, Boucher BL, Durieux-Smith A, Champagne SC, Morán LM. Frequency-specific audiometry using steady-state responses. Ear Hear 1996; 17: 81-96.

19. Pérez-Abalo MC, Savio G, Torres A, Martín V, Rodríguez E, Galán L. Steady state responses to multiple amplitude-modulated tones: an optimized method to test frequency-specific thresholds in hearing-impaired children and normal-hearing subjects. Ear Hear 2001; 22 : 200-11. 